\title{
The Dark Halo - Spheroid Conspiracy
}

\section{Rhea-Silvia Remus ${ }^{1,2}$, Andreas Burkert ${ }^{1,2}$, Klaus Dolag ${ }^{1,4}$, Peter H. Johansson ${ }^{3}$, Thorsten $\mathrm{Naab}^{4}$, Ludwig Oser ${ }^{4}$, and Jens Thomas ${ }^{2}$}

\author{
${ }^{1}$ Universitäts-Sternwarte München, Scheinerstr. 1, D-81679 München, Germany \\ ${ }^{2}$ MPI for Extraterrestrial Physics, PO Box 1312, D-85748 Garching, Germany \\ ${ }^{3}$ University of Helsinki, Gustaf Hällströmin katu 2a, FI-00014 Helsinki, Finland \\ ${ }^{4}$ MPI for Astrophysics, Karl-Schwarzschild-Str. 1, D-85748 Garching, Germany \\ email: rhea@usm.lmu.de
}

\begin{abstract}
Observational results from strong lensing and dynamical modeling indicate that the total density profiles of early-type galaxies are close to isothermal, i.e. $\rho_{\text {tot }} \propto r^{\gamma}$ with $\gamma \approx-2$. To understand the origin of this universal slope we study a set of simulated spheroids formed in cosmological hydrodynamical zoom-in simulations (see Oser et al. 2010 for more details). We find that the total stellar plus dark matter density profiles of all our simulations on average can be described by a power law with a slope of $\gamma \approx-2.1$, with a tendency towards steeper slopes for more compact, lower mass ellipticals, while the total intrinsic velocity dispersion is flat for all simulations, independent of the values of $\gamma$. Our results are in good agreement with observations of Coma cluster ellipticals (Thomas et al. 2007) and results from strong lensing (Sonnenfeld et al. 2012). We find that for $z \gtrsim 2$ the majority of the stellar build-up occurs through in-situ star formation, i.e. the gas falls to the center of the galaxy and forms stars, causing the galaxy to be more compact and thus the stellar component to be more dominant. As a result, the total density slopes at $z \approx 2$ are generally steeper (around $\gamma \approx-3$ ). Between $z=2$ and $z=0$ galaxies grow mostly through dry merging, with each merging event shifting the slope more towards $\gamma \approx-2$. We conclude from our simulations that the steepness of the slope of present day galaxies is a signature of the importance of mostly dry mergers in the formation of an elliptical, and suggest that all elliptical galaxies will with time end up in a configuration with a density slope of $\gamma \approx-2$. For a more detailed analysis with a larger sample of simulations see Remus et al. (2013).
\end{abstract}

Keywords. galaxies: kinematics and dynamics, structure, evolution - methods: numerical
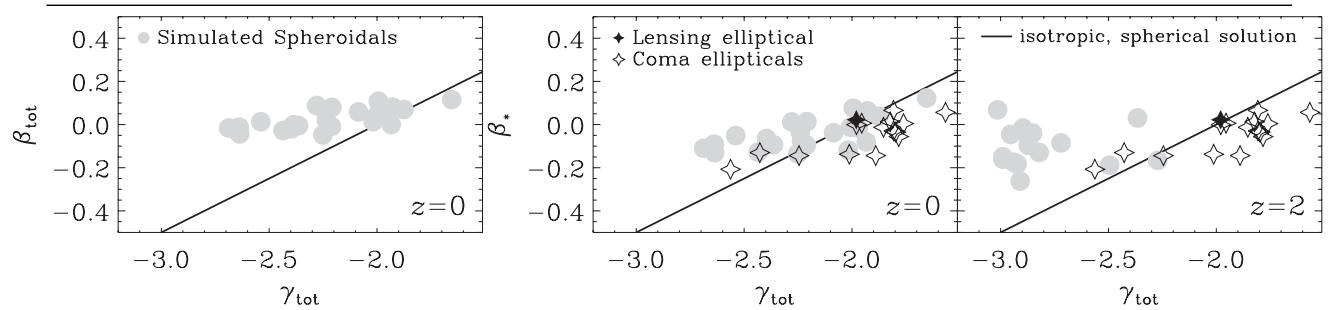

Figure 1. The Figure shows the slopes of the total (left panel) respectively the stellar (central and right panel) velocity dispersion profiles on the y-axis versus the slopes of the total density profiles on the $\mathrm{x}$-axis for the simulated ellipticals (light gray circles) at $z=0$ (left and central panel) and $z=2$ (right panel), the observations of the Coma cluster ellipticals (open black stars) and the strong lensing elliptical (filled black star).

\section{References}

Oser, L., Ostriker, J. P., Naab, T., Johansson, P. H., \& Burkert, A. 2010, ApJ, 725, 3212

Remus, R-S. et al. 2013, ApJ, 766, 71

Sonnenfeld, A., Treu, T., Gavazzi, R., Marshall, P. J., et al. 2012, ApJ, 752, 163

Thomas, J., Saglia, R. P., Bender, R., Thomas, D., et al. 2007, MNRAS, 382, 657 